\title{
Initial daytime and nighttime SOFDI observations of thermospheric winds from Fabry-Perot Doppler shift measurements of the 630-nm OI line-shape profile
}

\author{
A. J. Gerrard ${ }^{1}$ and J. W. Meriwether ${ }^{2}$ \\ ${ }^{1}$ Center for Solar-Terrestrial Research, Department of Physics, 323 Martin Luther King Boulevard, 101 Tiernan Hall, Newark, \\ NJ 07102-1982, USA \\ ${ }^{2}$ Department of Physics and Astronomy, Clemson University, Clemson, SC 29634-0978, USA
}

Received: 12 April 2011 - Revised: 26 July 2011 - Accepted: 9 August 2011 - Published: 2 September 2011

\begin{abstract}
In this paper we present both night and day thermospheric wind observations made with the Second-generation, Optimized, Fabry-Perot Doppler Imager (SOFDI), a novel triple-etalon Fabry-Perot interferometer (FPI) designed to make 24-h measurements of thermospheric winds from OI $630-\mathrm{nm}$ emission. These results were obtained from the northeastern United States and from under the magnetic equator at Huancayo, Peru and demonstrate the current instrument capability for measurements of Doppler shifts for either night or day. We found the uncertainties in the measurements agree with expected values based upon forward modeling calculations; nighttime wind components having an uncertainty of $\sim 20-\mathrm{m} \mathrm{s}^{-1}$ at 30-min resolution and daytime wind components having an uncertainty of $\sim 70$ $\mathrm{m} \mathrm{s}^{-1}$ at 20-min resolution. The nighttime uncertainties are typically larger than those seen with traditional single-etalon FPIs, which occur at the cost of being able to achieve daytime measurements. The thermospheric wind measurements from Huancayo replicate recently reported CHAMP zonal winds and are in disagreement with current empirical wind climatologies. In addition, we discuss the incorporation of how multiple point heads in the SOFDI instrument will allow for unique studies of gravity wave activity in future measurements.
\end{abstract}

Keywords. Ionosphere (Equatorial ionosphere)

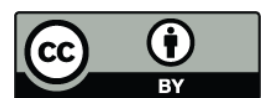

Correspondence to: A. J. Gerrard (gerrard@njit.edu)

\section{Introduction}

Understanding the development and evolution of localized spread-F is a problem of fundamental plasma physics as well as an important engineering issue (e.g., see Kelley, 2009, and references therein along with 13 consecutive years of papers from the Ionospheric Effects Symposium, Alexandria, VA, ies2011.com). A detailed study of such a phenomenon, along with a large host of other ionosphere/thermosphere issues (e.g., stormtime dynamics, nature of traveling ionospheric disturbances, solar cycle variability impacts on the thermosphere), requires measurements of the neutral background winds. However, such observations are sparse because the experimental challenges associated with such measurements are daunting, especially during the daytime. One instrument that has shown considerable effectiveness in the measurement of thermospheric winds is the Fabry-Perot interferometer (FPI). This instrument uses high spectral resolution measurements of the OI 630-nm spectral profile emission (originating from the broad oxygen layer in the thermosphere between $\sim 200-\mathrm{km}$ and $\sim 300-\mathrm{km}$ and peaking at $\sim 230-\mathrm{km}$ (Solomon and Abreu, 1989; Zhang and Shepherd, 2004)) in the lower thermosphere (Hernandez, 1986; Meriwether, 2006) to determine the line-of-sight Doppler shift and Doppler broadening for each direction observed.

However, most FPI measurements are only possible during nighttime conditions when there is no solar background obscuring the thermospheric emission line. Daytime observations, to date, are typically limited to emission intensities and temperatures (Barmore, 1977; Conde and Jacka, 1989; Pallamraju and Chakrabarti, 2005, 2006) or are extremely sparse (Cocks and Jacka, 1979). Using modern Fabry-Perot

Published by Copernicus Publications on behalf of the European Geosciences Union. 
technology, the Second-generation Optimized Fabry-Perot Doppler Imager (SOFDI) was constructed with the purpose of measuring continuous 24-h (i.e., nighttime and daytime) winds and temperatures from the upper mesosphere and lower thermosphere. This particular project was initially funded so that the 24-h ground-based wind measurements from SOFDI could be used to validate in-situ wind measurements from the Air Force Communications/Navigations Outage Forecasting System (C/NOFS) satellite, which is part of an intensive satellite and data assimilation/operational space weather modeling program that will attempt to predict the low latitude occurrence of equatorial spread-F (ESF). The SOFDI measurements may furthermore test recent theories that suggest that the timing of the afternoon reversal of the zonal wind from west to east is an important predictor of ESF development (Hysell and Kudeki, 2004; Kudeki et al., 2007).

In this paper we report on traditional nighttime and the first daytime results from SOFDI obtained while the instrument was undergoing testing in the northeastern United States as well as recent results from under the magnetic equator at Huancayo, Peru. These daytime results represent a major advance in ground-based daytime FPI operations. In Sect. 2 we describe the instrument design and in Sect. 3 we present thermospheric wind observations obtained for both day and night periods. We discuss these wind observations in Sect. 4 and present conclusions and future work in Sect. 5.

\section{Instrumentation}

SOFDI is a triple-etalon FPI capable of making wind and temperature measurements from the spectra obtained for a variety of upper atmospheric emission lines. The FabryPerot etalons, optics, housing, pressure/thermal and motion control systems of the SOFDI instrument were designed and integrated by Michigan Aerospace Corporation, Inc. The optical characteristics of the SOFDI etalons are identical to the etalons of the HRDI instrument flown on the UARS spacecraft (Skinner et al., 1987; Hays and HRDI Science Team, 1992) and consist of a high resolution (HRE, 1-cm fixed zerodur gap), a medium resolution (MRE, 0.186-cm piezoelectric tuned gap) and a low resolution (LRE, 0.024-cm piezoelectric tuned gap) etalon. Each fused-silica etalon is $\mathrm{ZnS}$ ThF4 coated, $8.8-\mathrm{cm}$ in effective diameter, is $86 \%$ reflective, and is mounted on motorized slides for insertion into the optical path upon command; thus, conversion from the daytime optical configuration to the nighttime setup or vice versa can be accomplished within a few minutes. In this paper we present only the results of thermospheric OI 630-nm emission wind measurements.

Light from each of the four independent, fully steerable, pointing mirror systems (i.e., SkyScanner pointing heads developed by KeoScientific, Inc.) is reflected into a $5.08-\mathrm{cm}$ $\left(2^{\prime \prime}\right)$ diameter telescope that focuses the light into a fiber op- tic bundle. The four fiber bundles, each representing an independent look direction, connect via a hermetic seal to a thermalized and pressurized optical chamber which contains all of the optics and other components of the SOFDI instrument. This pressurization and thermalization keeps the system stability to better than $5-\mathrm{m} \mathrm{s}^{-1}$ drift over $6 \mathrm{~h}$. The light from each fiber unit is collectively collimated and passed through an interference filter wheel, the etalon system, and then imaged onto a high quantum efficiency/low dark noise Andor CCD camera. A $1.0-\mathrm{nm}$ or a $0.5-\mathrm{nm}$ interference filter passband is used for either the nighttime or daytime OI observations, respectively.

Normally, each of the SkyScanners is sequentially pointed to an azimuth (east or north) at $60^{\circ}$ zenith angle. These would be followed by a vertical measurement to determine the zero-wind Doppler reference, and finally, in a direction toward the Sun for daytime measurements (the latter being required to account for the Doppler shifting of the solar spectrum introduced by the rotation of the earth and for accurate removal of the daytime continuum (Barmore, 1977)).

In Fig. 1 we show sample raw SOFDI data under nighttime, twilight, and daytime conditions. For nighttime operations only the HRE is placed in the optical path to image approximately 11 orders of each look direction onto the CCD. Each of the 11 orders represents an independent measurement of the wind and temperature in that particular look direction. For daytime operations, all three etalons are placed in the optical path so as to reduce solar contamination of the comparatively weak 630-nm dayglow line. Effectively, the LRE and MRE block 10 of the 11 orders of the HRE, allowing only 1 order to pass. Under normal operations, there would be three more data quadrants imaged on the CCD in Fig. 1, with each quadrant representing an independent look direction. The data in each quadrant is annularly integrated (within that quadrant) to form a spectrum.

For the data collected during nighttime conditions (i.e., when all 11 orders are recorded), each fringe in each quadrant is individually fitted with a Voigt profile (i.e., the resultant mathematical profile obtained from the convolution of a Lorentzian and a Gaussian profile, representing the instrument function and the thermal broadening OI linewidth, respectively) to determine the line center position and width. The measured line center for a particular fringe is compared to the corresponding line center of the fringe measured from the zenith-pointing direction and a Doppler shift, which is proportional to the line-of-sight thermospheric wind, is computed. By computing the Doppler shift (and Doppler broadening) for each fringe individually, any optical aberrations or illumination variation along the optical axis, i.e., out from the center of the CCD chip, are accounted for because such aberrations would similarly effect both fringes. So long as the vertical wind is much smaller than the horizontal wind, this vertical Doppler reference is valid. This approach is the one generally used in analyzing Fabry-Perot interferometer observations (Hernandez and Roble, 1979). The measured 


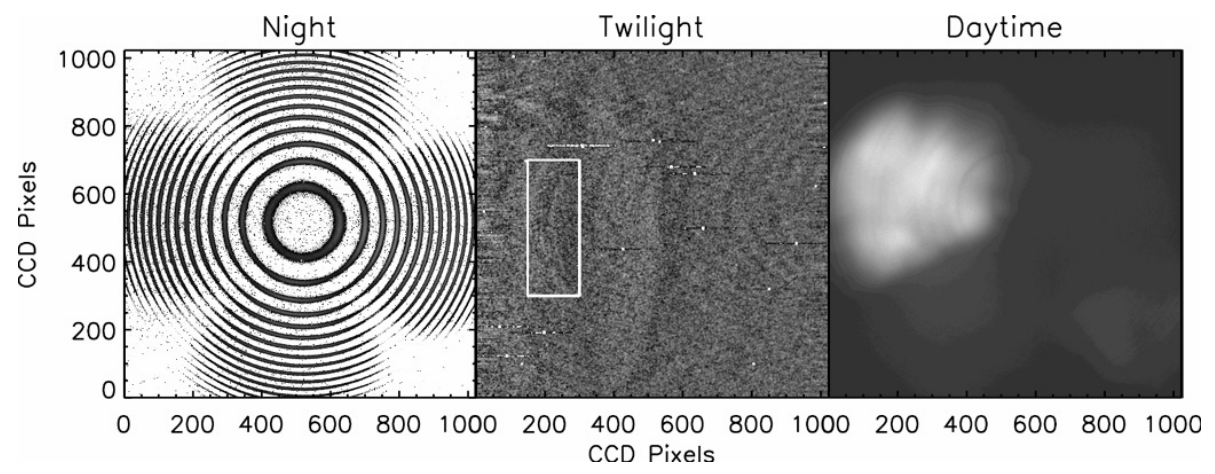

Fig. 1. Raw SOFDI data as imaged onto a CCD for three different observing conditions. left) Typical OI 630-nm nighttime data from all four field-of-views (hence four distinct quadrants) for just the high resolution etalon. Each fringe from each field-of-view yields an independent thermospheric wind measurement corresponding to that field-of-view. middle) Typical twilight data from one field-of-view for all three etalons in place. This image has been median filtered and smoothed to better display the OI fringe within the white bounding box. right) Raw daytime data showing the solar continuum and nearby absorption lines.

line width is used to compute the Doppler broadening and thus the species temperature.

This processing procedure for nighttime data has a number of caveats that need to be addressed. First, we approximate each fringe of the instrument function as a Lorentzian profile instead of a full Airy profile, which is the sum of overlapping Lorentzian functions. Essentially, we are neglecting the impact of the wings of the Lorentzian on adjacent fringes. Second, by processing and fitting each fringe separately we greatly minimize the contributions due to instrument etaloning (i.e., cavity reflections between the etalon and interference filter, or more generally between any optical surfaces) because the impact on a particular field-ofview fringe is the same as the impact on the vertical reference fringe. Similarly, any possible contamination from $\mathrm{OH}$ emission passing through the broader 1-nm interference filter is likewise removed in the nighttime winds. Third, due to $\mathrm{OH}$ contamination, we note that the measured OI temperatures will likely suffer a bias which is not accounted for herein. However, use of the HRE and MRE together, for example, in nighttime measurements can be used to remove this contamination bias in future measurements. Fourth, herein we utilize a Doppler reference determined by the "local" zenith measurement (i.e., the zenith measurement taken closest in time to the particular look direction). The use of this value as a Doppler reference can greatly impact the zonal and meridional wind measurements if there is a significant vertical wind. Use of the nightly mean vertical reference as the Doppler reference may be more adequate, but that too makes certain assumptions about the nature of the wind. Furthermore, the value of the "local" zenith wind is that each zonal or meridional measurement can be considered uncorrelated from consecutive measurements, thus allowing averaging of data and a simple reduction of the standard deviation of the mean, whereas a nighttime averaged vertical reference has a correlation structure that must be included in averaging consecutive data.
For the data collected during daytime conditions (i.e., when all 3 etalons are in the optical path and only 1 order is effectively recorded), we ratio the annularly integrated data in a particular field-of-view with the corresponding quadrant data obtained from a solar spectrum (Noxon et al., 1979; Tepley et al., 1981). This ratio removes the solar continuum and leaves only atmospheric emission structure; namely the OI emission and contributions due to the Ring Effect (i.e., the filling of Fraunhoffer valleys due to atmospheric scattering (Barmore, 1975; Pallamraju et al., 2002)). The dayglow OI fringe is located very close to where one of the nighttime fringes would be located and is fit with a Voigt profile, while the Ring continuum is simultaneously fit with a broader Gaussian-shape and its contribution is removed from the Voigt fit as seen in Fig. 2. The OI line center computed from this fit is then compared to the OI line center computed from the fit of the fringe obtained from the vertical field-ofview, and again the Doppler shift is computed assuming the vertical wind is much smaller than the horizontal wind.

There are two notable caveats that need to be mentioned when computing the daytime winds. First, any spectral structure in the solar spectrum can have a profound impact on both the signal-to-noise ratio and the bias of the wind determination. The presence of tropospheric cirrus clouds, in particular, can substantially limit the observational data window. A zenith pointing, wide field-of-view web-camera is mounted in the SOFDI trailer to assist in the detection of such clouds. Second, at times the Ring Effect is not necessarily Gaussian in shape and there can be $\sim 20-\mathrm{m} \mathrm{s}^{-1}$ impacts, obtained by using various fitting functions/methods, on the estimated winds. Furthermore, the Ring Effect will have an impact on determining the line emission width and thus daytime temperature measurements, which to date, have not been reliably computed. 


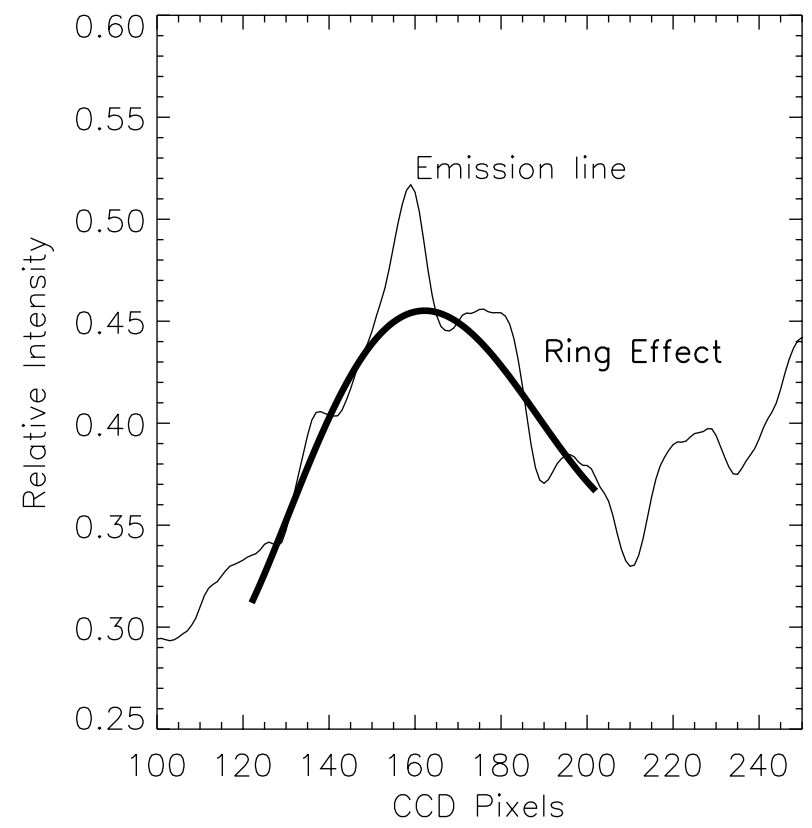

Fig. 2. Example of the dayglow 630-nm OI emission, after the sky spectrum is divided by the solar spectrum. The thick black line traces the component due to the Ring Effect (i.e., the line sketches the Ring Effect profile), and the OI emission line is seen atop of the Ring Effect continuum. This OI emission is then fit with a Voigt profile.

\section{SOFDI measurements from one pointing head}

Hereafter we report on the results obtained from SOFDI utilizing only one of the four pointing heads and thus represent only a fourth of SOFDI's functionality. This allows the reader to scale the reported measurements as they deem appropriate when all four pointing-heads are used, for example, to increase temporal resolution, reduce measurement uncertainty, or configured for unique observation schemes of, for example, gravity waves (discussed below).

From June 2006 to March 2008 SOFDI was located in Steuben Valley in upstate New York $\left(43.3^{\circ} \mathrm{N}, 75.3^{\circ} \mathrm{W}, 250\right.$ $\mathrm{m}$ altitude). Throughout this period SOFDI had been making regular nighttime thermospheric OI 630-nm wind measurements and sporadic daytime wind measurements. In Fig. 3 we show one sample period of daytime wind data collected from the use of one SkyScanner pointing head on 21 March 2008 , during a low Kp period (less than $\sim 3$ ) that characteristic of the most recent solar minimum. Each nominal data realization was taken over a 5-min period, with a full wind vector obtained very 20 -min. Wind measurement uncertainties are $\sim 50-\mathrm{m} \mathrm{s}^{-1}$ at 20 -min resolution. Also plotted in Fig. 3 are the corresponding zonal and meridional winds as computed from the Horizontal Wind Model-2007 (HWM07) (Drob et al., 2008; Emmert et al., 2008) for reference. These sample wind measurements are of the same general magnitude as the empirical model wind values. There is a clear

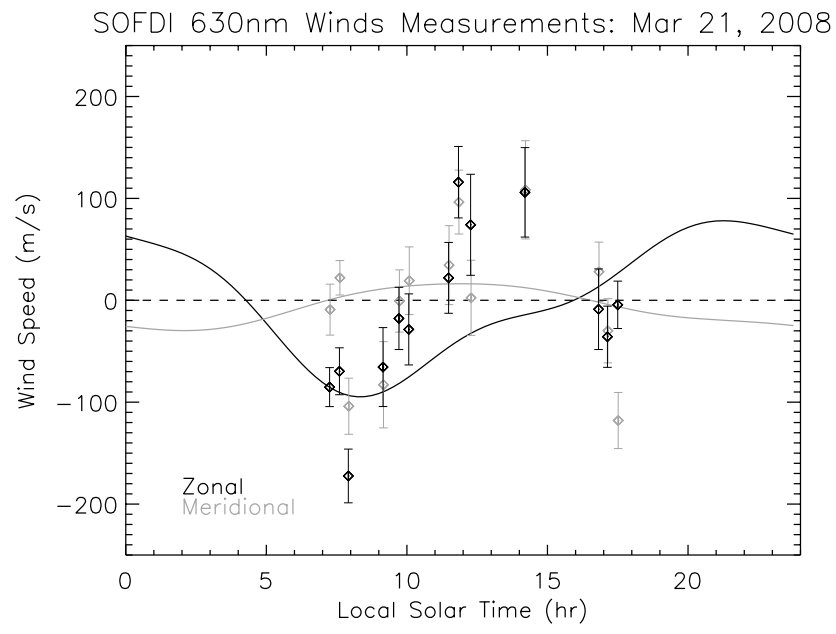

Fig. 3. Daytime OI 630-nm thermospheric winds, represented by diamond points with 1-sigma uncertainty bars, as measured from one field-of-view by SOFDI in Steuben Valley, NY from 28 March 2008. The thin lines are from the HWM07 empirical model. Black data points/lines represent the zonal wind (positive values are eastward) while gray data points/lines represent the meridional wind (positive values are northward).

difference, however, in the structure of the wind response throughout the day. For example, though it appears that the zonal wind reversal from a westward to eastward phase initiates at approximately the same time ( $\sim 08: 00 \mathrm{LST})$, the timescale of the reversal occurs earlier on this particular day than what the climatological model shows.

In 2009 SOFDI was transported to Huancayo, Peru $\left(12.0^{\circ} \mathrm{S}, 284.8^{\circ} \mathrm{E}\right.$, geographic, $0.6^{\circ} \mathrm{N}, 356.2^{\circ} \mathrm{E}$, geomagnetic, $3.2-\mathrm{km}$ altitude) to make thermospheric wind observations in support of the C/NOFS program. Nighttime data was successfully recorded in June 2010 and both daytime and nighttime data in August 2010. A sample of data taken with one SkyScanner pointing head from August 2010 is shown in Figs. 4 and 5, where we show the measured winds and nighttime temperatures, respectively.

In Fig. 4, each nominal data realization for the nighttime wind data was taken over a $10-\mathrm{min}$ period, with a full wind vector obtained every $30-\mathrm{min}$, while for the daytime wind data each nominal data realization was taken over a 5-min period, with a full wind vector obtained every $15-\mathrm{min}$. The gaps in the daytime data are due to internal system checks and calibration, and represent an extremely conservative/cautious mode of operation. Each of the daytime measurements has an uncertainty of $\sim 70-\mathrm{m} \mathrm{s}^{-1}$ at the 20-min resolution, while each of the nighttime measurements has an uncertainty of $\sim 20-\mathrm{m} \mathrm{s}^{-1}$ at 30 -min resolution. HWM07 wind values are shown for reference. The daytime data presented here have higher uncertainties (as compared to the data shown in Fig. 3) due to non-optimal alignment of the triple etalon system. Nighttime data was collected before 10:00 LST and after 

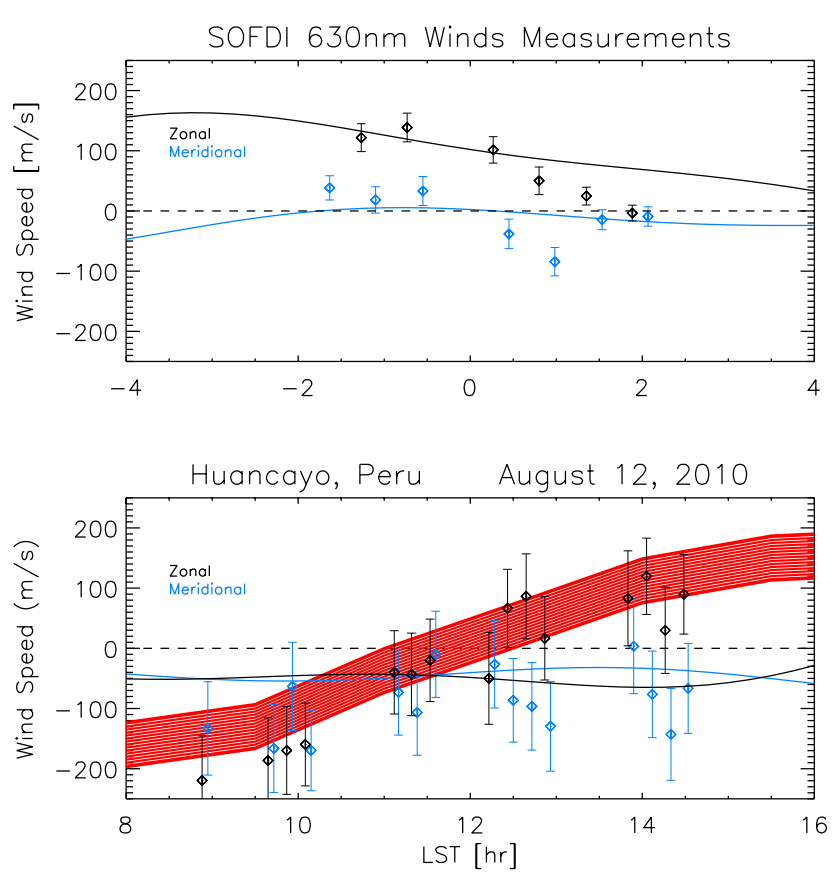

Fig. 4. Top: nighttime OI 630-nm thermospheric winds, represented by diamond points with 1-sigma uncertainty bars, obtained by SOFDI in Huancayo, Peru from the night of 11 August to 12 August 2010. The thin lines are from the HWM07 empirical model. Black data points/lines represent the zonal wind (positive values are eastward) while light blue data points/lines represent the meridional wind (positive values are northward). Bottom: daytime OI 630-nm thermospheric winds as measured from one field-of-view by SOFDI on 12 August 2010, presented in the same manner. The red bar represents the 1-sigma range of geophysical variability of climatological CHAMP winds during equinox at low Kp and low F10.7 values (Liu et al., 2006).

02:00 LST, but the signal levels were too low to determine a wind measurement with this level of uncertainty.

In Fig. 5, the nighttime temperature measurements from the different look directions were not separated and are instead plotted together, showing the degree of variability based on pointing-head look direction. As compared to concurrent FPI measurements from the Jicamarca FPI, these temperatures appear to be $\sim 50-\mathrm{K}$ too high, likely the result of the aforementioned $\mathrm{OH}$ contamination. Outside of the bias, these temperature measurements have an uncertainty of $\sim 40-\mathrm{K}$.

For completeness, we note that during periods of very weak nighttime $\mathrm{OI}$ emission (i.e., as were typical during the March 2008 period from the mid-latitude trough) removal of the instrument function from the measured OI spectrum is difficult because of the low ionospheric temperatures (and hence narrow Doppler linewidths). Consequently, we did not show SOFDI nighttime winds or temperatures from the 2008 data here due to the weak and narrow 630-nm spectral emission.

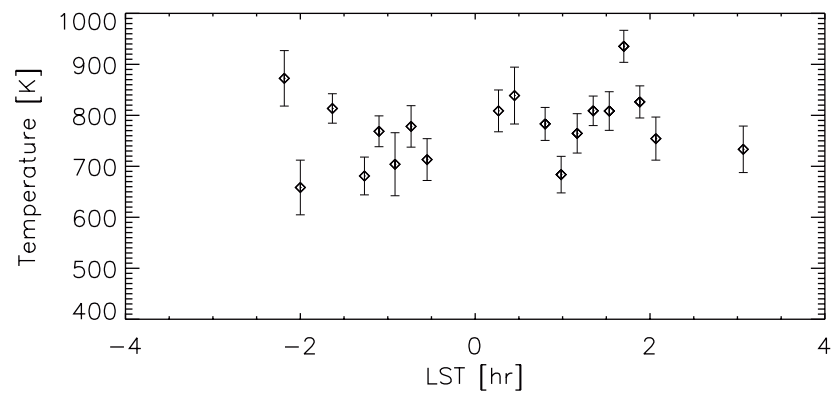

Fig. 5. Nighttime OI 630-nm thermospheric temperatures obtained by SOFDI from the night of 11 August to 12 August 2010. Data from the different pointing-head look directions was not separated and demonstrates the spatial variability of the temperature measurements.

\section{Discussions}

The sample data presented here in Figs. 3, 4, and 5 are typical daytime and nighttime data that can be obtained with the SOFDI instrument at this time. To date, the SOFDI thermospheric wind measurements made in upstate NY are consistent with both climatological Millstone Hill FPI thermospheric wind data (Emmert et al., 2003) and with synoptic Millstone Hill Incoherent Scatter Radar meridional wind estimates (Gerrard et al., 2009). This statement is based on 15 nights of data from Fall of 2005, 6 samples of daytime and nighttime data from mid-March 2008, and sporadic data samples taken throughout 2006 and 2007. The daytime data from upstate NY is, at this time, relatively sparse due to data contamination of thin cirrus clouds. However, we are currently reanalyzing this data utilizing a new inversion scheme in the attempt to remove this cloud influence.

Nighttime data from Huancayo have been compared to FPI data from Jicamarca (located at the new FPI observatory, MeriHill) under similar observational conditions and compare remarkably well, with the exception that SOFDI temperatures appear to be $\sim 50-\mathrm{K}$ higher in value. In summer 2011 we plan on obtaining and comparing thermospheric temperatures obtained with just the HRE and with the HRE-MRE combination in an effort to quantify the $\mathrm{OH}$ contamination issue. This result will be valuable to other FPIs used in the community that use a $1-\mathrm{cm}$ spacer gap.

This sample of daytime data from Huancayo was consistent with other SOFDI daytime measurements made over three different days in August 2010. We note that the uncertainty bars presented here are likely overestimated, as the individual data values shown in Fig. 4 do not share or indicate the same variance (and would justify further averaging of the independent data points). Moreover, the consistent discrepancy between the SOFDI winds and HWM07 initially gave us large concern for our inversion methods, until we compared these data to recent equatorial zonal wind data from the CHAMP satellite (Liu et al., 2006, 2009), also shown 

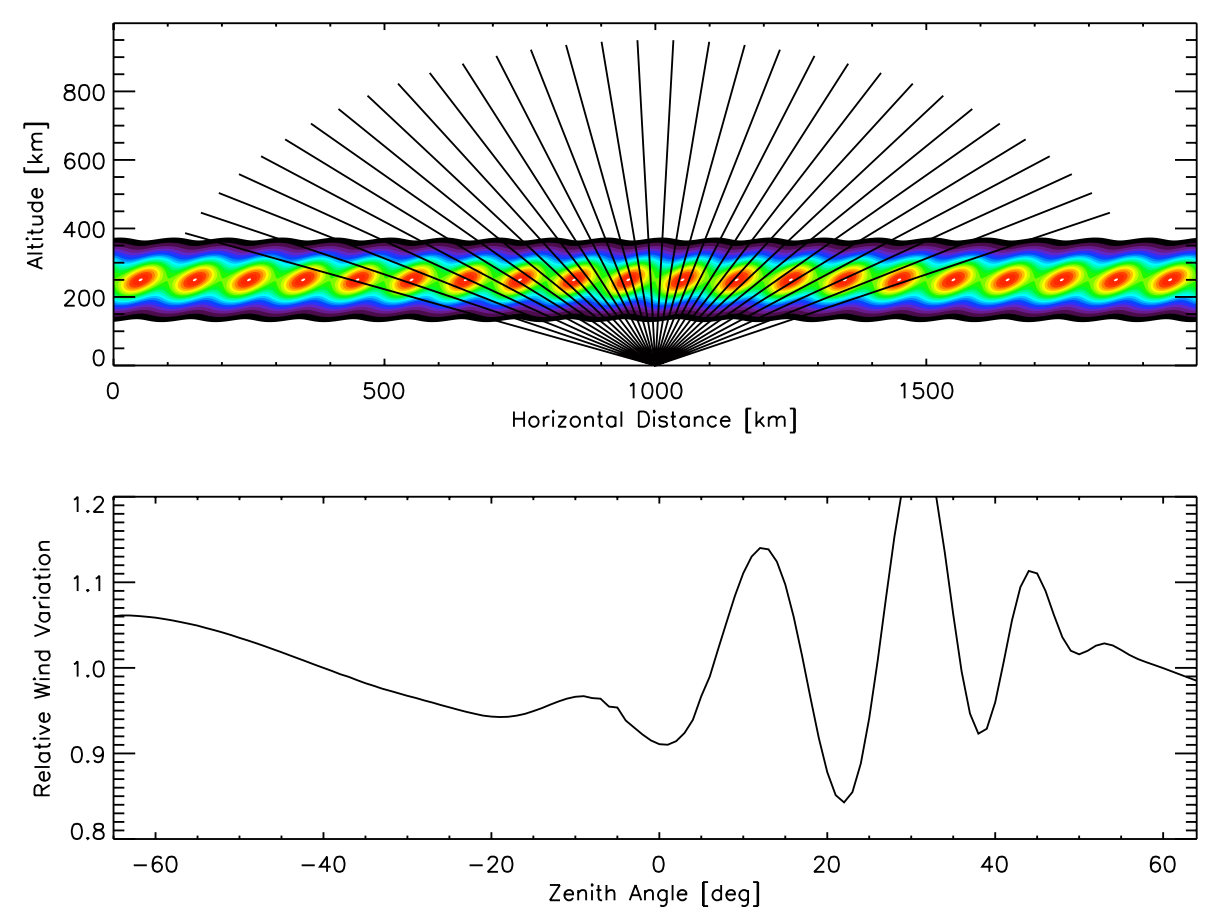

Fig. 6. Based on the gravity wave structures presented in Oliver et al. (1994), a model reconstruction of the measured horizontal wind field as a function of line-of-sight. (top) A spatial construct of the viewing geometry, with horizontal distance in the x-axis and altitude in the z-axis. The sum of the mean wind field and the gravity wave wind field $(\sim 200-\mathrm{km}$ vertical wavelength, ground-based period of $\sim 1$-h, amplitude of $\sim 20 \%$ of the mean background wind), weighted by the vertical emission intensity profile, is depicted in color with each line representing a different FPI line-of-sight. (bottom) The relative variation of the measured wind speed as a function of the line-of-sight zenith angle (i.e., a value of " 1 " indicates a measurement that is equal to the true mean background wind). Only at large or negative (i.e., line-of-sights opposing the gravity wave phase structure) zenith angles would the impact of the gravity wave variation on the measured horizontal wind be averaged out over the line-of-sight.

in Fig. 4 for reference. Both CHAMP and SOFDI daytime wind data compare very well and are in disagreement with HWM07 (and previous versions) winds. Such differences between observations and HWM07 have been observed in other studies, e.g., in (Kosch et al., 2000), in Brazilian FPI observations by Meriwether and Makela (J. Makela, personal communications, 2011), etc. However, we note that there are a large number of issues with that need to be addressed in comparing empirical climatologies with "semi-synoptic" observations (D. Drob, personal communications, 2011). Because the daytime HWM07 equatorial winds are highly influenced by both the DE2-spacecraft wind measurements from the early 1980s and the optical WINDII measurements from the 1990s, there may be some potentially interesting issues that might need to be resolved influencing the geophysics between the 1980s-1990s and the 2000s.

Furthermore, we note that both CHAMP and SOFDI consistently see a wind reversal from westward to eastward around local noon, as opposed to the local evening. This appears to coincide with a thermally direct neutral wind circulation, but the lack of daytime temperatures prevents us from verifying this. The timing of this reversal is vitally important to current ESF formation theories and ongoing C/NOFS op- erational forecasts. With the initiation of another solar cycle, concurrent C/NOFS neutral wind observations and SOFDI observations will provide an exciting opportunity to investigate the role of the neutral winds on ESF.

Finally, we would like to comment on a 3-day set of data collected from 30 October to 1 November 2008 (not shown) which showed dramatic variability in the measured winds that were not consistent with typical FPI observations. To date we have not been able to dismiss this data on account of experimental errors (e.g., etalon drift, cloud influence). The only experimental differences between this particular set of observations and all other observations were that (1) the data were taken in New Jersey, closer to the Atlantic coast, and (2) the measurements were made at a $45^{\circ}$ zenith angle. These measurements showed wind values that varied much more than the wind values of March, at times reaching $\sim 250-\mathrm{m} \mathrm{s}^{-1}$. Though such a wind speed itself isn't unusual (Emmert et al., 2003), the wind shears, observed during both day and night conditions, and at times exceeding $10-\mathrm{m} \mathrm{s}^{-1} \mathrm{~min}^{-1}$, are dramatic. Furthermore, though the measured winds show the same general trend/phase as the empirical model, the differences between model and observation often exceed $100-\mathrm{m} \mathrm{s}^{-1}$. Nighttime FPI data from 
Millstone Hill (obtained from the NCAR-CEDAR Database) show such wind shears and deviations from models occasionally, but they seem quite infrequent.

Close inspection of the October 2008 data indicate that the measured zonal and meridional winds show a $\sim 3-5 \mathrm{~h}$ periodicity, potentially indicating a wave structure with a smaller spatial scale than that of an atmospheric tide. This periodicity, along with the higher viewing angle, leads one to suspect that we are observing the apparent modulation of the assumed "mean horizontal wind speed" due to the propagation of any such gravity wave structure. For example, in Fig. 6 we show model results of the measured horizontal wind as a function of zenith angle/line-of-sight for an upwardly propagating gravity wave with a $\sim 200-\mathrm{km}$ vertical wavelength, a ground-based period of $\sim 1-\mathrm{h}$, and an amplitude of $\sim 20 \%$ of the mean background wind (i.e., if time was to step forward, the gravity wave phase lines would progress in a "downward diagonal" to the right). Such a thermospheric gravity wave is inferred from structures presented in, for example, Oliver et al. (1994). We see that the measured horizontal wind, when the instrument line-of-sight is aligned with the inclination angle of the gravity wave (i.e., the angle of the gravity wave phase structure with respect to the horizontal), can be modulated by as much as the ratio of the gravity wave amplitude to the mean horizontal wind speed, depending on the actual observational slant path through the gravity wave structure. As the gravity wave propagates upwards, the assumed measured horizontal wind speed would be similarly modulated in time. When the line-of-sight is opposed to the phase structure of the gravity wave, the gravity wave undulations are spatially averaged out over the slant path, resulting in a more accurate measurement of the mean horizontal wind speed.

Most FPI measurements are taken at very high zenith angle and thus tend to average out any potential gravity wave influence because gravity waves at these altitudes tend to be propagating more vertically (i.e., at high inclination angles). Though this yields a better measurement of the horizontal wind speed, it removes any insight into gravity waves at these altitudes.

Because the SOFDI instrument has four independent SkyScanner pointing heads, one can envision one possible observational scheme in which two heads point to the east at $30^{\circ}$ and $60^{\circ}$ zenith angles and the other two heads point to the west, also at $30^{\circ}$ and $60^{\circ}$ zenith angles, and simultaneously measure the zonal thermospheric wind field. This would give both the background/mean zonal wind and, assuming a gravity wave is present and propagating near a $30^{\circ}$ inclination angle, the zonal gravity wave amplitude and zonal inclination angle. Moving all 4 pointing heads to corresponding meridional positions would then yield the background meridional wind, the meridional gravity wave amplitude, and the meridional inclination angle. A related observational scheme would be to scan in azimuth the four pointing heads, each set at a particular zenith angle, in order to located gravity wave structure that is not necessarily at a $30^{\circ}$ inclination angle. As far as we know, SOFDI is the only instrument that is currently capable of making such unique gravity wave measurements both at night and during the day.

\section{Conclusions}

In this paper we have presented daytime and nighttime thermospheric winds as measured from OI 630-nm emission by the SOFDI instrument. We have demonstrated that measurement uncertainties are within previously reported ranges and are well within observed geophysical variability. We note that the daytime wind measurements presented herein represent substantial progress in FPI instrumental design and validates the SOFDI approach to nighttime and daytime wind measurements. Though the uncertainties in Figs. 3, 4, and 5 may seem larger than those associated with other nighttimeonly FPI instruments, they are the result of the trade-offs made to obtain daytime measurements and are nonetheless acceptable for a large variety of thermospheric studies.

At this time, all four of SOFDI's pointing heads are operating and collecting daytime and nighttime OI data at Huancayo, Peru. The SOFDI data collection system and etalon tuning algorithm are robust enough to operate unattended, but sudden power failure or the presence of thin cirrus clouds can impact operations. That is, the SOFDI control system requires additional engineering support to enable truly autonomous operation. Current work involves making this operation procedure more robust, addressing the $\mathrm{OH}$ contamination issue in nighttime temperatures, and the continued collection of equatorial thermospheric winds in an effort to clarify the underlaying physics of ESF generation.

Acknowledgements. The authors would like to thank the personnel of Michigan Aerospace Corporation, Inc. for their substantial contributions on the SOFDI project. We specifically thank Paul Hays, Jane Pavlich, Scott Lindemann, Charles Richey, James Borck, Greg Wassick, Pete Tchoryk, Carl Nardell, and Michael Dehring for their invaluable assistance through the entire construction, integration, and testing process. We also thank the Geophysical Institute of Peru (IGP) for providing logistical support while in Peru, namely Jorge Chau, Ronald Woodman, Erick Vidal Safor, and Oscar Veliz. Similarly, we thank the Gerrard family for their logistical support on this project while SOFDI was located in upstate New York. This work was supported by grants from the National Science Foundation (NSF-ATM-0735452 and NSF-ATM-0734241) and from the Air Force Office of Scientific Research (AFOSRFA9550-08-1-0087).

Topical Editor K. Kauristie thanks M. Kosch and another anonymous referee for their help in evaluating this paper.

\section{References}

Barmore, F. E.: The Filling-In of Fraunhofer Lines in the Day Sky., J. Atmos. Sci., 32, 1489-1493, doi:10.1175/15200469(1975)032<1489:TFIOFL>2.0.CO;2, 1975. 
Barmore, F. E.: High resolution observations of the $6300 \AA$ oxygen line in the day airglow, Planet. Space Sci., 25, 185-191, doi:10.1016/0032-0633(77)90023-X, 1977.

Cocks, T. D. and Jacka, F.: Daytime thermospheric temperatures, wind velocities and emission intensities derived from ground based observations of the O I lambda $630 \mathrm{~nm}$ airglow line profile, J. Atmos. Terr. Phys., 41, 409-415, 1979.

Conde, M. and Jacka, F.: Analysis of day-time observations of the $630 \mathrm{~nm}$ thermospheric emission over Mawson, Antarctica, ANARE Res. Notes, 69, 125-138, 1989.

Drob, D. P., Emmert, J. T., Crowley, G., Picone, J. M., Shepherd, G. G., Skinner, W., Hays, P., Niciejewski, R. J., Larsen, M., She, C. Y., Meriwether, J. W., Hernandez, G., Jarvis, M. J., Sipler, D. P., Tepley, C. A., O’Brien, M. S., Bowman, J. R., Wu, Q., Murayama, Y., Kawamura, S., Reid, I. M., and Vincent, R. A.: An empirical model of the Earth's horizontal wind fields: HWM07, J. Geophys. Res., 113, 12304, doi:10.1029/2008JA013668, 2008.

Emmert, J. T., Fejer, B. G., and Sipler, D. P.: Climatology and latitudinal gradients of quiet time thermospheric neutral winds over Millstone Hill from Fabry-Perot interferometer measurements, J. Geophys. Res., 108, 1196, doi:10.1029/2002JA009765, 2003.

Emmert, J. T., Drob, D. P., Shepherd, G. G., Hernandez, G., Jarvis, M. J., Meriwether, J. W., Niciejewski, R. J., Sipler, D. P., and Tepley, C. A.: DWM07 global empirical model of upper thermospheric storm-induced disturbance winds, J. Geophys. Res., 113, 11319, doi:10.1029/2008JA013541, 2008.

Gerrard, A. J., Meriwether, J. W., Goncharenko, L. P., Hedden, R. B., and Kelley, M.: Comparisons of neutral thermospheric winds as measured by SOFDI and the Millstone Hill Incoherent Scatter Radar, AGU Spring Meeting Abstracts, pp. A22+, 2009.

Hays, P. B. and HRDI Science Team: Remote sensing of mesospheric winds with the high-resolution doppler imager, Planet. Space Sci., 40, 1599-1606, doi:10.1016/0032-0633(92)90119-9, 1992.

Hernandez, G.: Fabry-Perot Interferometers, Cambridge Studies in Modern Optics, Cambridge: University Press, 1986, 1986.

Hernandez, G. and Roble, R. G.: Thermospheric dynamics investigations with very high resolution spectrometers, Appl. Optics, 18, 3376-3385, 1979.

Hysell, D. L. and Kudeki, E.: Collisional shear instability in the equatorial F region ionosphere, J. Geophys. Res., 109, 11301, doi:10.1029/2004JA010636, 2004.

Kelley, M. C.: The Earth's Ionosphere: Plasma Physics and Electrodynamics, 2nd edition, Elsevier, London, 2009.

Kosch, M. J., Ishii, M., Nozawa, S., Rees, D., Cierpka, K., Kohsiek, A., Schlegel, K., Fujii, R., Hagfors, T., Fuller-Rowell, T. J., and Lathuillere, C.: A comparison of thermospheric winds and temperatures from Fabry-Perot interferometer and EISCAT radar measurements with models, Adv. Space Res., 26(6), 979-984, 2000 .
Kudeki, E., Akgiray, A., Milla, M., Chau, J. L., and Hysell, D. L.: Equatorial spread-F initiation: Post-sunset vortex, thermospheric winds, gravity waves, J. Atmos. Solar-Terr. Phys., 69, 24162427, doi:10.1016/j.jastp.2007.04.012, 2007.

Liu, H., Lühr, H., Watanabe, S., Köhler, W., Henize, V., and Visser, P.: Zonal winds in the equatorial upper thermosphere: Decomposing the solar flux, geomagnetic activity, and seasonal dependencies, J. Geophys. Res., 111, A07307, doi:10.1029/2005JA011415, 2006.

Liu, H., Watanabe, S., and Kondo, T.: Fast thermospheric wind jet at the Earth's dip equator, Geophys. Res. Lett., 36, L08103, doi:10.1029/2009GL037377, 2009.

Meriwether, J. W.: Studies of thermospheric dynamics with a Fabry-Perot interferometer network: A review, J. Atmos. SolarTerr. Phys., 68, 1576-1589, 2006.

Noxon, J. F., Whipple, Jr., E. C., and Hyde, R. S.: Stratospheric NO2. I - Observational method and behavior at mid-latitude, J. Geophys. Res., 84, 5047-5065, doi:10.1029/JC084iC08p05047, 1979.

Oliver, W. L., Fukao, S., Yamamoto, Y., Takami, T., Yamanaka, M. D., Yamamoto, M., Nakamura, T., and Tsuda, T.: Middle and upper atmosphere radar observations of ionospheric density gradients produced by gravity wave packets, J. Geophys. Res., 99, 6321-6329, doi:10.1029/94JA00171, 1994.

Pallamraju, D. and Chakrabarti, S.: First ground-based measurements of OI $6300 \AA$ daytime aurora over Boston in response to the 30 October 2003 geomagnetic storm, Geophys. Res. Lett., 32, 3, doi:10.1029/2004GL021417, 2005.

Pallamraju, D. and Chakrabarti, S.: Contributions of imaging Echelle spectrographs to daytime optical aeronomy, J. Atmos. Solar-Terr. Phys., 68, 1459-1471, doi:10.1016/j.jastp.2005.05.013, 2006.

Pallamraju, D., Baumgardner, J., and Chakrabarti, S.: HIRISE: a ground-based high-resolution imaging spectrograph using echelle grating for measuring daytime airglow/auroral emissions, J. Atmos. Solar-Terr. Phys., 64, 1581-1587, doi:10.1016/S13646826(02)00095-0, 2002.

Skinner, W. R., Hays, P. B., and Abreu, V. J.: Optimization of a triple etalon interferometer, Appl. Optics, 26, 2817-2827, 1987.

Solomon, S. C. and Abreu, V. J.: The $630 \mathrm{~nm}$ dayglow, J. Geophys. Res., 94, 6817-6824, doi:10.1029/JA094iA06p06817, 1989.

Tepley, C. A., Meriwether Jr., J. W., Walker, J. C. G., and Mathews, J. D.: Observations of neutral iron emission in twilight spectra, J. Geophys. Res., 86, 4831-4835, doi:10.1029/JA086iA06p04831, 1981.

Zhang, S. P. and Shepherd, G. G.: Solar influence on the $\mathrm{O}\left({ }^{1} D\right)$ dayglow emission rate: Global-scale measurements by WINDII on UARS, Geophys. Res. Lett., 31, L07804, doi:10.1029/2004GL019447, 2004. 